\title{
Near-Earth objects from the cometary flux
}

\author{
Vacheslav Emel'yanenko \\ Institute of Astronomy RAS \\ e-mail: vvemel@inasan.ru
}

\begin{abstract}
We analyze the orbital distribution of objects captured to near-Earth space from the flux of comets coming from the outer Solar system. For this purpose, we use the model of the cometary cloud developed earlier (Emelfyanenko, Asher, Bailey, 2007). This model is consistent with the broad dynamical characteristics of observed near-parabolic comets, short-period comets, Centaurs and high-eccentricity trans-Neptunian objects. We show that the observed distributions of both large and small near-Earth objects are different from the modeled distribution formed dynamically by the action of planetary perturbations. In particular, while the distributions of arguments of perihelion for observed Jupiter-family comets and modeled cometary asteroids follow a sinusoidal law with pronounced maxima around 0 and 180 degrees, it is not the case for observed cometary asteroids of any size. We conclude that there exist many unobserved extinct short-period comets among near-Earth objects of various sizes.
\end{abstract}

